\title{
A New Approach to General Interpolation Formulae for Bivariate Interpolation
}

\author{
Le Zou' ${ }^{1}$ and Shuo Tang ${ }^{2}$ \\ ${ }^{1}$ Key Lab of Network and Intelligent Information Processing, Hefei University, Hefei 230601, China \\ ${ }^{2}$ Department of Mathematics, Hefei University of Technology, Hefei 230039, China \\ Correspondence should be addressed to Le Zou; zoule1983@163.com
}

Received 6 February 2014; Revised 14 April 2014; Accepted 7 May 2014; Published 25 June 2014

Academic Editor: Sofiya Ostrovska

Copyright (c) 2014 L. Zou and S. Tang. This is an open access article distributed under the Creative Commons Attribution License, which permits unrestricted use, distribution, and reproduction in any medium, provided the original work is properly cited.

\begin{abstract}
General interpolation formulae for bivariate interpolation are established by introducing multiple parameters, which are extensions and improvements of those studied by Tan and Fang. The general interpolation formulae include general interpolation formulae of symmetric branched continued fraction, general interpolation formulae of univariate and bivariate interpolation, univariate block based blending rational interpolation, bivariate block based blending rational interpolation and their dual schemes, and some interpolation form studied by many scholars in recent years. We discuss the interpolation theorem, algorithms, dual interpolation, and special cases and give many kinds of interpolation scheme. Numerical examples are given to show the effectiveness of the method.
\end{abstract}

\section{Introduction}

Newton interpolation and Thiele-type continued fractions interpolation may be the favored linear interpolation and nonlinear interpolation [1]. Symmetric branched continued fraction is a bivariate continued fractions interpolation scheme discussed by Cuyt and Verdonk [2,3], Kučminskaja [4], and Murphy and O'Donohoe [5]. In recent years, Kuchmins'ka and Vozna [6, 7], Pahirya [8], Zhao [9], and Wang [10] studied some new kinds of symmetric blending rational interpolation. Wang and Qian studied bivariate polynomial interpolation and continued fractions interpolation over ortho-triples [11]. Zhao and Tan studied block based Newtonlike blending rational interpolation [12] and block based Thiele-like blending rational interpolation [13]. The general frames of interpolation problem have been widely studied. Kahng showed the generalizations of univariate Newton's method and applied it to the approximation problems in 1967 [14]; Kahng described a class of interpolation functions and showed the explicit method of osculatory interpolation with a function in the class in 1969 [15]. In 1999, Tan and Fang [1] studied several general frames for bivariate interpolation which include many classical interpolation schemes; Tan also discussed the more general interpolation grids [16]. Recently, Tang and Zou [17] have improved and extended the general frames studied by Tan and Fang by introducing multiple parameters, so that the new frames can be used to deal with the interpolation problems where inverse differences are nonexistent or unattainable points occur. The general form of block based bivariate blending rational interpolation with the error estimation is established by introducing two parameters [18]; four different block based interpolations are included. Then an efficient algorithm for computing bivariate lacunary rational interpolation is constructed based on block based bivariate blending rational interpolation. One of authors constructs the frames of symmetry interpolation [19] and general structures of one and two variable interpolation function without depending on the existence of divided difference or inverse differences, and he also discusses the block based osculatory interpolation in one variable case [20].

Our contribution in this paper is to obtain a new type of general interpolation formulae for bivariate interpolation by introducing multiple parameters, which includes general interpolation formula of symmetric branched continued fraction, general interpolation formulae of univariate and bivariate interpolation, univariate block based blending rational 
interpolation, bivariate block based blending rational interpolation and their dual schemes, and some new interpolation scheme studied by many scholars in recent years. The organization of the paper is as follows. In Section 2 we discuss the interpolation theorem, algorithms, dual interpolation, and special cases of general interpolation formulae of symmetric interpolation. The interpolation theorem, algorithms, dual interpolation, and special cases of the general interpolation formulae of block based univariate and bivariate interpolation are discussed in Section 3. Numerical examples are given to show the effectiveness of the method in Section 4.

\section{General Interpolation Formulae of Symmetric Interpolation}

Given a set of real points $\Pi_{n, m}=\left\{\left(x_{i}, y_{j}\right) \mid i=0,1, \ldots, n, j=\right.$ $0,1, \ldots, m\} \subset[a, b] \times[c, d] \subset R^{2}$ and a bivariate function $f(x, y)$ defined in a domain $[a, b] \times[c, d]$.

Notation 1. Let

$$
N=\max \left\{i \mid\left(x_{i}, y_{i}\right) \in \Pi_{n, m}\right\}
$$

Now we construct a function

$$
\begin{aligned}
& Q(x, y)=f_{0}\left(A_{0}(x, y)+S_{0}(x, y)\right. \\
& \times f_{1}\left(A_{1}(x, y)+S_{1}(x, y)\right. \\
& \times f_{2}\left(A_{2}(x, y)+\cdots+S_{N-1}(x, y)\right. \\
&\left.\left.\left.\quad \times f_{N}\left(A_{N}(x, y)\right) \cdots\right)\right)\right),
\end{aligned}
$$

by constructing different $A_{i}(x, y)$; then, $Q(x, y)$ can be changed into general frame of symmetric interpolation [19], general frame of block based univariate interpolation $[17,20]$, general frame of block based bivariate interpolation $[17,20]$, and so on.

If we choose $A_{i}(x, y)$ as follows in formula (2):

$$
\begin{aligned}
A_{i}(x, y)=f_{i, i}\left(a_{i, i}(x, y)+\right. & g_{i}(x) \\
\times f_{i+1, i}( & a_{i+1, i}(x, y)+\cdots+g_{n-1}(x) \\
& \left.\left.\quad \times f_{n, i}\left(a_{n, i}(x, y)\right) \cdots\right)\right) \\
+h_{i}(y) f_{i, i+1}( & a_{i, i+1}(x, y)+\cdots \\
& \left.+h_{m-1}(y) f_{i, m}\left(a_{i, m}(x, y)\right) \cdots\right),
\end{aligned}
$$

where $S_{i}(x, y)=\left(x-x_{i}\right)\left(y-y_{i}\right), a_{i, j}(x, y)$ are constants, $g_{i}(x)=x-x_{i}, h_{i}(y)=y-y_{i}$, then $Q(x, y)$ is a general interpolation formula of symmetric interpolation.

We cite a theorem and one can prove (2), (3) are a general interpolation formula of symmetric interpolation and satisfy interpolation conditions.
Notation 2. Let

$$
\begin{aligned}
& h(A)=\{h(x) \mid x \in A\}, \\
& R(h) \text { : range of } h(x) .
\end{aligned}
$$

Theorem 1 (see [15]). Given a function $y(x)$ continuous in a finite interval $[a, b]$ and $n+1$ points $x_{i}$ with $a \leqslant x_{0}<x_{1}<\cdots<$ $x_{n} \leqslant b$, there exists a unique set of parameters $a_{0}, a_{1}, \ldots, a_{n}$ such that the interpolation function

$$
Q(x)=f_{0}\left(a_{0}+g_{0}(x) f_{1}\left(a_{1}+\cdots+g_{n-1}(x) f_{n}\left(a_{n}\right), \ldots\right)\right),
$$

satisfying

$$
Q\left(x_{i}\right)=y\left(x_{i}\right), \quad i=0,1 \ldots, n,
$$

and $Q(x)$ is continuous if

(a) $f_{i}$ is continuous and strictly monotone in $(-\infty,+\infty)$ and the range of $f_{i}(x)$ covers $(-\infty,+\infty), i=$ $1,2, \ldots, n$;

(b) $f_{0}$ is continuous and its inverse function $f_{0}^{-1}$ exists in $R\left(f_{0}\right)$ and $R\left(f_{0}\right) \supset y([a, b])$;

(c) the functions $g_{j}(x), j=0,1, \ldots, n-1$, are continuous in $[a, b]$ and

$$
g_{j}(x) \begin{cases}=0, & x=x_{j} \\ \neq 0, & x>x_{j}\end{cases}
$$

When the above conditions are satisfied, the parameters are determined from the following equations in sequence: $a_{0}=f_{0}^{-1}\left(Q\left(x_{0}\right)\right)$ is determined from $Q\left(x_{0}\right)=f_{0}\left(a_{0}\right), a_{1}=$ $f_{1}^{-1}\left(\left(f_{0}^{-1}\left(Q\left(x_{1}\right)\right)-a_{0}\right) / g_{0}\left(x_{1}\right)\right)$ is found from $Q\left(x_{1}\right)=f_{0}\left(a_{0}+\right.$ $\left.g_{0}\left(x_{1}\right) f_{1}\left(a_{1}\right)\right)$, and so on.

The conditions on the functions $f_{i}, f_{i, j}, g_{i}, h_{i, j}$ for the existence of unique parameters $a_{i, j}(i=0,1, \ldots, n, j=$ $0,1, \ldots, m)$ are given next using the following notations.

Notation 3. Let

$$
h(A, B)=\{h(x, y) \mid(x, y) \in[a, b] \times[c, d]\}
$$$$
S(h) \text { : range of } h(x, y) \text {. }
$$

Theorem 2. Given a function $f(x, y)$ continuous in $[a, b] \times$ $[c, d]$ and $(n+1) \times(m+1)$ points $\left(x_{i}, y_{j}\right)$ such that

$a \leqslant x_{0}<x_{1}<\cdots<x_{n} \leqslant b ; \quad c \leqslant y_{0}<y_{1}<\cdots<y_{m} \leqslant d$, 
then there exists a unique set of parameters $a_{0,0}, a_{0,1}, \ldots, a_{N, m}$ for the interpolation function

$$
\begin{aligned}
& Q(x, y)=f_{0}\left(A_{0}(x, y)+S_{0}(x, y)\right. \\
& \times f_{1}\left(A_{1}(x, y)+\right. S_{1}(x, y) \\
& \times f_{2}\left(A_{2}(x, y)+\cdots+S_{N-1}(x, y)\right. \\
&\left.\left.\left.\quad \times f_{N}\left(A_{N}(x, y)\right) \cdots\right)\right)\right),
\end{aligned}
$$

$$
\begin{aligned}
A_{i}(x, y)=f_{i, i}\left(a_{i, i}(x, y)+\right. & g_{i}(x) \\
\times f_{i+1, i}( & a_{i+1, i}(x, y)+\cdots+g_{n-1}(x) \\
\times & \left.\left.f_{n, i}\left(a_{n, i}(x, y)\right) \cdots\right)\right) \\
+h_{i}(y) f_{i, i+1}( & a_{i, i+1}(x, y)+\cdots+h_{m-1}(y) \\
& \left.\times f_{i, m}\left(a_{i, m}(x, y)\right) \cdots\right),
\end{aligned}
$$

satisfying

$$
Q\left(x_{i}, y_{j}\right)=f\left(x_{i}, y_{j}\right), \quad i=0,1, \ldots, n, j=0,1, \ldots, m,
$$

if

(a) $f_{i}, f_{i, j}$ are continuous and strictly monotone in their domain of definitions and their ranges are $(-\infty,+\infty)$, $i=1,2, \ldots, n, j=0,1, \ldots, m$,

(b) $f_{0}, f_{i, 0}$ are continuous and their inverse functions $f_{0}^{-1}$, $f_{i, 0}^{-1}$ exist in $S\left(f_{0}\right), S\left(f_{i, 0}\right)$, respectively, and $S\left(f_{0}\right)$ ว $f\left([a, b], y_{0}\right), S\left(f_{i, 0}\right) \supset f\left(x_{i},[c, d]\right)$,

(c) functions $g_{i}(x), i=0,1, \ldots, n-1 ; h_{i, j}(y), i=$ $0,1, \ldots, n, j=0,1, \ldots, m-1$, are continuous in $[a, b]$, $[c, d]$, respectively, and

$$
g_{i}(x)=\left\{\begin{array} { l l } 
{ = 0 , } & { x = x _ { i } ; } \\
{ \neq 0 , } & { x > x _ { i } ; }
\end{array} \quad h _ { i , j } ( y ) \left\{\begin{array}{cc}
=0, & y=y_{j} \\
\neq 0, & y>y_{j}
\end{array}\right.\right.
$$

Proof. If $x=x_{0}$, then

$$
\begin{aligned}
& Q\left(x_{0}, y\right) \\
& \begin{aligned}
=f_{0}\left(f _ { 0 , 0 } \left(a_{0,0}+h_{0}(y) f_{0,1}(\right.\right. & a_{0,1}+\cdots+h_{m-1}(y) \\
& \left.\left.\left.\times f_{0, m}\left(a_{0, m}\right) \cdots\right)\right)\right) ;
\end{aligned}
\end{aligned}
$$

this is just the univariate structure; from Theorem 1, we can get $Q\left(x_{0}, y_{j}\right)=f\left(x_{0}, y_{j}\right)$, and $Q\left(x_{0}, y\right)$ is continuous.

Similarly, if $y=y_{0}$,

$$
\begin{aligned}
& Q\left(x, y_{0}\right) \\
& =f_{0}\left(f _ { 0 , 0 } \left(a_{0,0}+g_{0}(x) f_{1,0}\left(a_{1,0}+\cdots+g_{n-1}(x)\right.\right.\right. \\
& \left.\left.\left.\times f_{n, 0}\left(a_{n, 0}\right) \cdots\right)\right)\right) \text {; }
\end{aligned}
$$

we can get $Q\left(x, y_{0}\right)=f\left(x, y_{0}\right)$ easily, and $Q\left(x, y_{0}\right)$ is continuous. Similarly, if $x=x_{1}$,

$$
\begin{aligned}
& Q\left(x_{1}, y\right)=f_{0}\left(A_{0}\left(x_{1}, y\right)+S_{0}\left(x_{1}, y\right) f_{1}\left(A_{1}\left(x_{1}, y\right)\right)\right), \\
& \begin{aligned}
& A_{0}\left(x_{1}, y\right) \\
&=f_{0,0}\left(a_{0,0}+g_{0}\left(x_{1}\right) f_{1,0}\left(a_{1,0}\right)+h_{0}(y)\right. \\
& \quad\left.\quad \times f_{0,1}\left(a_{0,1}+\cdots+h_{m-1}(y) f_{0, m}\left(a_{0, m}\right) \cdots\right)\right),
\end{aligned} \\
& \begin{aligned}
A_{1}\left(x_{1}, y\right) \\
=f_{1,1}\left(a_{1,1}+h_{1}(y)\right. \\
\left.\quad \times f_{1,2}\left(a_{1,2}+\cdots+h_{m-1}(y) f_{1, m}\left(a_{1, m}\right) \cdots\right)\right),
\end{aligned}
\end{aligned}
$$

and if $y=y_{1}$,

$$
\begin{aligned}
& Q\left(x, y_{1}\right)=f_{0}\left(A_{0}\left(x, y_{1}\right)+S_{0}\left(x, y_{1}\right) f_{1}\left(A_{1}\left(x, y_{1}\right)\right)\right), \\
& \begin{array}{l}
A_{0}\left(x, y_{1}\right), \\
\quad=f_{0,0}\left(a_{0,0}+g_{0}(x) f_{1,0}\left(a_{1,0}+\cdots+g_{n-1}(x) f_{n, 0}\left(a_{n, 0}\right)\right)\right. \\
\left.\quad+h_{0}\left(y_{1}\right) f_{0,1}\left(a_{0,1}\right)\right),
\end{array} \\
& \begin{array}{l}
A_{1}\left(x, y_{1}\right) \\
=f_{1,1}\left(a_{1,1}+g_{1}(x) f_{2,1}\left(a_{2,1}+\cdots+g_{n-1}(x) f_{n, 1}\left(a_{n, 1}\right)\right)\right) .
\end{array}
\end{aligned}
$$

We repeat the above process similarly, and finally we can obtain $Q\left(x_{n}, y_{j}\right)=f\left(x_{n}, y_{j}\right)$, and $Q\left(x_{n}, y\right)$ is continuous. When the above conditions are satisfied, the parameters $a_{0,0}, a_{0,1}, \ldots, a_{n, m}$ are determined from the following equations in sequence.

From $Q\left(x_{0}, y_{0}\right)=f_{0}\left(f_{0,0}\left(a_{0,0}\right)\right)$, we can get

$$
a_{0,0}=f_{0,0}^{-1}\left(f_{0}^{-1}\left(\mathrm{Q}\left(x_{0}, y_{0}\right)\right)\right) ;
$$

from $Q\left(x_{0}, y_{1}\right)=f_{0}\left(f_{0,0}\left(a_{0,0}+h_{0}\left(y_{1}\right) f_{0,1}\left(a_{0,1}\right)\right)\right)$, we can get

$$
a_{0,1}=f_{0,1}^{-1} \frac{f_{0,0}^{-1}\left(f_{0}^{-1}\left(Q\left(x_{0}, y_{1}\right)\right)\right)-a_{0,0}}{h_{0}\left(y_{1}\right)} ;
$$

from $Q\left(x_{0}, y_{2}\right)=f_{0}\left(f_{0,0}\left(a_{0,0}+h_{0}\left(y_{2}\right) f_{0,1}\left(a_{0,1}+h_{1}\left(y_{2}\right)\right.\right.\right.$ $\left.\left.f_{0,2}\left(a_{0,2}\right)\right)\right)$ ), we can get

$a_{0,2}$

$$
\begin{gathered}
=f_{0,2}^{-1}\left(f_{0,1}^{-1}\left(\frac{f_{0,0}^{-1}\left(f_{0}^{-1}\left(Q\left(x_{0}, y_{2}\right)\right)\right)-a_{0,0}}{h_{0}\left(y_{2}\right)}-a_{1,1}\right)\right. \\
\left.\times\left(h_{1}\left(y_{2}\right)\right)^{-1}\right) ;
\end{gathered}
$$

finally, we can obtain the parameters

$$
a_{0, j}, \quad j=0,1, \ldots, m
$$


from $Q\left(x_{1}, y_{0}\right)=f_{0}\left(f_{0,0}\left(a_{0,0}+g_{0}\left(x_{1}\right) f_{1,0}\left(a_{1,0}\right)\right)\right)$, we can get

$$
a_{1,0}=f_{1,0}^{-1} \frac{f_{0,0}^{-1}\left(f_{0}^{-1}\left(Q\left(x_{1}, y_{0}\right)\right)\right)-a_{0,0}}{g_{0}\left(x_{1}\right)} ;
$$

from $Q\left(x_{2}, y_{0}\right)=f_{0}\left(f_{0,0}\left(a_{0,0}+g_{0}\left(x_{2}\right) f_{1,0}\left(a_{1,0}+g_{1}\left(x_{2}\right)\right.\right.\right.$ $\left.\left.f_{2,0}\left(a_{2,0}\right)\right)\right)$ ), we can get

$a_{2,0}$

$$
\begin{gathered}
=f_{1,0}^{-1}\left(f_{1,0}^{-1}\left(\frac{f_{0,0}^{-1}\left(f_{0}^{-1}\left(Q\left(x_{2}, y_{0}\right)\right)\right)-a_{0,0}}{g_{0}\left(x_{2}\right)}-a_{1,0}\right)\right. \\
\left.\times\left(g_{1}\left(x_{2}\right)\right)^{-1}\right) .
\end{gathered}
$$

Using the induction method, finally, we can obtain all the parameters

$$
a_{i, j}, \quad i=0,1, \ldots, n, j=0,1, \ldots, m \text {. }
$$

Thus, this proves the theorem.

2.1. Special Cases. Some of the special cases of the above general interpolation formula of bivariate symmetry interpolation function are shown below.

(1) If $f_{i}(x)=x, i=0,1, \ldots, N, f_{i, j}(x)=x, S_{i}(x, y)=$ $\left(x-x_{i}\right)\left(y-y_{i}\right), a_{i, j}(x, y)$ are constants, $g_{i}(x)=x-x_{i}$, $h_{i}(y)=y-y_{i}, i=0,1, \ldots, n, j=0,1, \ldots, m$, then $Q(x, y)$ is bivariate Newton interpolation polynomial [16].

(2) If $f_{0}(x)=x, f_{i}(x)=1 / x, f_{i, i}(x)=x, f_{i, j}(x)=1 / x$, $i \neq j, S_{i}(x, y)=\left(x-x_{i}\right)\left(y-y_{i}\right), a_{i, j}(x, y)$ are constants, $g_{i}(x)=x-x_{i}, h_{i}(y)=y-y_{i}, i=0,1, \ldots, n$, $j=0,1, \ldots, m$, then $Q(x, y)$ is bivariate symmetric continued fractions interpolation studied by many authors $[2-5,8]$.

(3) If $f_{0}(x)=x, f_{i}(x)=1 / x, f_{i, i}(x)=x, f_{i, j}(x)=1 / x$, $i \neq j, S_{i}(x, y)=\left(x-x_{i}\right)\left(y-y_{i}\right), a_{i, j}(x, y)$ are constants, $g_{i}(x)=x-x_{i}, h_{i}(y)=y-y_{i}, i=0,1, \ldots, n, j=$ $0,1, \ldots, m$, then $Q(x, y)$ is Stieltjes-Newton rational interpolation studied by Wang [10]; Zhao and Tan [9] also studied it and its limiting case.

(4) If $f_{0}(x)=x, f_{i}(x)=1 / x, f_{i, i}(x)=x, f_{i, j}(x)=x^{(-1)^{i+1}}$, $i>j, f_{i, j}(x)=x^{(-1)^{j+1}}, i<j, S_{i}(x, y)=\left(x-x_{i}\right)(y-$ $\left.y_{i}\right), a_{i, j}(x, y)$ are constants, $g_{i}(x)=x-x_{i}, h_{i}(y)=$ $y-y_{i}, i=0,1, \ldots, n, j=0,1, \ldots, m$, then $Q(x, y)$ is Newton-Thiele-like interpolation formula studied by Kuchmins'ka and Vozna [6, 7].

(5) If $f_{i}(x)=x, f_{i, i}(x)=x, f_{i, j}(x)=x^{(-1)^{i+1}}, i>j$, $f_{i, j}(x)=x^{(-1)^{j+1}}, i<j, S_{i}(x, y)=\left(x-x_{i}\right)\left(y-y_{i}\right)$, $a_{i, j}(x, y)$ are constants, $g_{i}(x)=x-x_{i}, h_{i}(y)=$ $y-y_{i}, i=0,1, \ldots, n, j=0,1, \ldots, m$, then $Q(x, y)$ is symmetric Newton associated continued fraction blending rational interpolation.
(6) If $f_{0}(x)=x, f_{i}(x)=x^{\delta}, f_{i, i}(x)=x, f_{i, j}(x)=x^{\delta}, \delta=$ 1 or $-1, g_{i}(x)=x-x_{i}, h_{i}(y)=y-y_{i}, i=0,1, \ldots, n$, $j=0,1, \ldots, m$, then $Q(x, y)$ is the general frame of symmetry interpolation studied by Tan and Fang [1].

(7) If $f_{0}(x)=x, f_{i}(x)=x^{(-1)^{i+1}}, f_{i, j}(x)=x^{(-1)^{i+1}}$, $g_{i}(x)=x-x_{i}, h_{i}(y)=y-y_{i}, i=0,1, \ldots, n$, $j=0,1, \ldots, m$, then $Q(x, y)$ is the general frame of symmetry interpolation studied by Zou and Tang [19].

(8) Suppose that the fixed points are arranged in groups of threes, which form $n+1$ L-like configurations. If $f_{0}(x)=x, f_{i}(x)=x, 1 \leq i \leq N-1, f_{i, i}(x)=x$, $f_{i, i+1}(x)=f_{i+1, i}(x)=x, f_{i, j}(x)=f_{j, i}(x)=x, j \geq i+2$, $g_{i}(x)=x-x_{2 i}, h_{i}(y)=y-y_{2 i}, a_{i, i+s}(x)=a_{2 i, 2 i+s}, 1 \leq$ $s \leq n-i, a_{i+t, i}(x)=a_{2 i+t, 2 i}, 1 \leq t \leq m-i, i=0,1, \ldots, n$; $j=0,1, \cdots, m$, then $Q(x, y)$ is bivariate polynomial interpolation over ortho-triples studied by Salzer [21].

(9) Suppose that the fixed points are arranged in groups of threes, which form $n+1$ L-like configurations. If $f_{0}(x)=x, f_{i}(x)=1 / x, 1 \leq i \leq N-1, f_{i, i}(x)=$ $x, f_{i, i+1}(x)=f_{i+1, i}(x)=1 / x, f_{i, j}(x)=f_{j, i}(x)=x$, $j \geq i+2, g_{i}(x)=x-x_{2 i}, h_{i}(y)=y-y_{2 i}, a_{i, i+s}(x)=$ $a_{2 i, 2 i+s}, 1 \leq s \leq n-i, a_{i+t, i}(x)=a_{2 i+t, 2 i}, 1 \leq t \leq$ $m-i, i=0,1, \ldots, n, j=0,1, \ldots, m$, then $Q(x, y)$ is bivariate continued fraction interpolation over orthotriples studied by Wang and Qian [11].

(10) If $f_{0}(x)=x, f_{i}(x)=f_{i, i}(x)=x^{(-1)^{i+1}}, f_{i, j}(x)=$ $x^{(-1)^{i+1}}, i>j, f_{i, j}(x)=x^{(-1)^{j+1}}, i<j, S_{i}(x, y)=$ $\left(x-x_{i}\right)\left(y-y_{i}\right), a_{i, j}(x, y)$ are constants, $g_{i}(x)=x-x_{i}$, $h_{i}(y)=y-y_{i}, i=0,1, \ldots, n, j=0,1, \ldots, m$, then $Q(x, y)$ is a new type of symmetric blending rational interpolation.

(11) If $f_{0}(x)=x, f_{i}(x)=f_{i, i}(x)=x, f_{i, j}(x)=x$, $i>j, f_{i, j}(x)=x^{(-1)^{j+1}}, i<j, S_{i}(x, y)=(x-$ $\left.x_{i}\right)\left(y-y_{i}\right), a_{i, j}(x, y)$ are constants, $g_{i}(x)=x-x_{i}$, $h_{i}(y)=y-y_{i}, i=0,1, \ldots, n, j=0,1, \ldots, m$, then $Q(x, y)$ is a new type of symmetric blending rational interpolation.

(12) If $f_{i}(x)=x, f_{i, i}(x)=x, f_{i, j}(x)=x, i>j$, $f_{i, j}(x)=x^{(-1)}, i<j, S_{i}(x, y)=\left(x-x_{i}\right)\left(y-y_{i}\right)$, $a_{i, j}(x, y)$ are constants, $g_{i}(x)=x-x_{i}, h_{i}(y)=y-y_{i}$, $i=0,1, \ldots, n, j=0,1, \ldots, m$, then $Q(x, y)$ is a new type of symmetric blending rational interpolation.

(13) If $f_{0}(x)=x, f_{i}(x)=x^{-1}, f_{i, i}(x)=x, f_{i, j}(x)=x, i>$ $j, f_{i, j}(x)=x^{(-1)}, i<j, S_{i}(x, y)=\left(x-x_{i}\right)\left(y-y_{i}\right)$, $a_{i, j}(x, y)$ are constants, $g_{i}(x)=x-x_{i}, h_{i}(y)=y-y_{i}$, $i=0,1, \ldots, n, j=0,1, \ldots, m$, then $Q(x, y)$ is a new type of symmetric blending rational interpolation.

(14) If $f_{0}(x)=x, f_{i}(x)=x^{(-1)^{i+1}}, f_{i, i}(x)=x, f_{i, j}(x)=x$, $i>j, f_{i, j}(x)=x^{(-1)}, i<j, S_{i}(x, y)=\left(x-x_{i}\right)\left(y-y_{i}\right)$, $a_{i, j}(x, y)$ are constants, $g_{i}(x)=x-x_{i}, h_{i}(y)=y-y_{i}$, $i=0,1, \ldots, n, j=0,1, \ldots, m$, then $Q(x, y)$ is a new type of symmetric blending rational interpolation. 
(15) If $i(\bmod 3)=0, f_{i}(x)=1 / x$, otherwise, $f_{i}(x)=x$, $f_{i, i}(x)=x, f_{i, j}(x)=x, i>j, f_{i, j}(x)=x^{(-1)}, i<j$, $S_{i}(x, y)=\left(x-x_{i}\right)\left(y-y_{i}\right), a_{i, j}(x, y)$ are constants, $g_{i}(x)=x-x_{i}, h_{i}(y)=y-y_{i}, i=0,1, \ldots, n, j=$ $0,1, \ldots, m$, then $Q(x, y)$ is a new type of symmetric blending rational interpolation.

Furthermore, one can get more symmetric blending rational interpolations via choosing $f_{i}(x), f_{i, j}(x)$ appropriately, for example, some new schemes given in the paper [19]. It is not difficult to generalize the general structure in this paper to higher dimensions or a vector-valued case or a matrix-valued case $[16,22,23]$.

\section{General Interpolation Formulae for Block Based Bivariate Interpolation}

Now we consider the general interpolation formulae of the following scheme; we divide $\prod_{n, m}=\left\{\left(x_{i}, y_{j}\right) \quad \mid i=\right.$ $0,1, \ldots, n, j=0,1, \ldots, m\}$ into $(u+1) \times(v+1)$ subsets; namely,

$$
\begin{array}{r}
\prod_{n, m}^{s, t}=\left\{\left(x_{i}, y_{j}\right) \mid c_{s} \leq i \leq d_{s}, h_{t} \leq j \leq r_{t}\right\}, \\
s=0,1, \ldots, u, t=0,1, \ldots, v .
\end{array}
$$

The subsets may be achieved by reordering the interpolation points if necessary.

If we choose $A_{i}(x, y)$ as follows in formula (2):

$$
\begin{aligned}
& A_{i}(x, y)=f_{i, 0}\left(a_{i, 0}(x, y)\right.+g_{0}(x) \\
& \times f_{1, i}\left(a_{1, i}(x, y)+\cdots+g_{u-1}(x)\right. \\
&\left.\left.\quad \times f_{u, i}\left(a_{u, i}(x, y)\right) \ldots\right)\right) \\
&+h_{0}(y) f_{i, 1}\left(a_{i, 1}(x, y)+\cdots+h_{v-1}(y)\right. \\
&\left.\quad \times f_{i, v}\left(a_{i, v}(x, y)\right) \ldots\right),
\end{aligned}
$$

then $Q^{\prime}(x, y)$ is a general interpolation formula of block based bivariate interpolation.

Theorem 3. Given a function $f(x, y)$ continuous in $[a, b] \times$ $[c, d]$ and $(n+1) \times(m+1)$ points $\left(x_{i}, y_{j}\right)(i=0,1, \cdots<n, j=$ $0,1, \ldots, m)$ such that

$$
a \leqslant x_{0}<x_{1}<\cdots<x_{n} \leqslant b ; \quad c \leqslant y_{0}<y_{1}<\cdots<y_{m} \leqslant d,
$$

then there exists a unique set of parameters $a_{0,0}, a_{0,1}, \ldots, a_{n, m}$ for the interpolation function

$$
\begin{aligned}
& \begin{aligned}
& Q^{\prime}(x, y)=f_{0}\left(A_{0}(x, y)+\right. S_{0}(x, y) \\
& \times f_{1}\left(A_{1}(x, y)+S_{1}(x, y)\right. \\
& \times f_{2}\left(A_{2}(x, y)+\cdots+S_{N-1}(x, y)\right. \\
&\left.\left.\left.\times f_{N}\left(A_{N}(x, y)\right) \cdots\right)\right)\right) \\
& A_{i}(x, y)=f_{i, 0}\left(a_{i, 0}(x, y)+g_{0}(x)\right. \\
& \times f_{1, i}\left(a_{1, i}(x, y)+\cdots+g_{u-1}(x)\right. \\
&\left.\left.\times f_{u, i}\left(a_{u, i}(x, y)\right) \ldots\right)\right) \\
&+h_{0}(y) f_{i, 1}\left(a_{i, 1}(x, y)+\cdots+h_{v-1}(y)\right. \\
&\left.\times f_{i, v}\left(a_{i, v}(x, y)\right) \ldots\right)
\end{aligned}
\end{aligned}
$$

satisfying

$$
Q^{\prime}\left(x_{i}, y_{j}\right)=f\left(x_{i}, y_{j}\right), \quad i=0,1, \ldots, n, j=0,1, \ldots, m,
$$

if

(a) $f_{i}, f_{i, j}$ are continuous and strictly monotone in their domain of definitions and their ranges are $(-\infty,+\infty)$, $i=1,2, \ldots, n ; j=0,1, \ldots, m$;

(b) $f_{0}, f_{i, 0}$ are continuous and their inverse functions $f_{0}^{-1}$, $f_{i, 0}^{-1}$ exist in $S\left(f_{0}\right), S\left(f_{i, 0}\right)$, respectively, and $S\left(f_{0}\right)$ > $f\left([a, b], y_{0}\right), S\left(f_{i, 0}\right) \supset f\left(x_{i},[c, d]\right) ;$

(c) functions $g_{i}(x), i=0,1, \ldots, n-1 ; h_{i, j}(y), i=$ $0,1, \ldots, n, j=0,1, \ldots, m-1$, are continuous in $[a, b]$, $[c, d]$, respectively, and

$$
g_{i}(x)\left\{\begin{array} { l l } 
{ = 0 , } & { x = x _ { i } ; } \\
{ \neq 0 , } & { x > x _ { i } ; }
\end{array} \quad h _ { i , j } ( y ) \left\{\begin{array}{ll}
=0 & y=y_{j} \\
\neq 0 & y>y_{j}
\end{array}\right.\right.
$$

We can prove the previous theorem similarly.

3.1. General Interpolation Formulae for Block Based Univariate Interpolation. If we choose the parameters in formulae (2), (25) as follows, we can get general interpolation formulae for block based univariate interpolation.

(1) If we choose $f_{i, 1}(x) \equiv 0, f_{1}(x) \equiv 0, g_{s}(x)=\prod_{i=c_{s}}^{d_{s}}(x-$ $\left.x_{i}\right), i=0,1, \ldots, n, s=0,1, \ldots, u-1$, we can get

$$
\begin{aligned}
\widetilde{Q}(x, y)=f_{0} & \left(f _ { 0 , 0 } \left(a_{0,0}(x, y)+g_{0}(x)\right.\right. \\
& \times f_{1,0}\left(a_{1,0}(x, y)+\cdots+g_{u-1}(x)\right. \\
& \left.\left.\left.\times f_{u, 0}\left(a_{u, 0}(x, y)\right) \cdots\right)\right)\right),
\end{aligned}
$$


where $a_{i, j}(x, y)=a_{i}(x)$ are univariate interpolating polynomial, rational interpolation, the Hermite interpolating polynomial, or Salzer-type osculatory rational interpolation. Then $\widetilde{Q}(x, y)$ is a general interpolation formula of block based univariate interpolation.

(2) If we choose $f_{1, i}(x) \equiv 0, f_{1}(x) \equiv 0, h_{t}(y)=g_{s}(x)=$ $\prod_{i=c_{s}}^{d_{s}}\left(x-x_{i}\right), s=0,1, \ldots, u-1$, we can get

$$
\begin{aligned}
& \bar{Q}(x, y)=f_{0}\left(f _ { 0 , 0 } \left(a_{0,0}(x, y)\right.\right.+h_{0}(y) \\
& \times f_{0,1}\left(a_{0,1}(x, y)+\cdots+h_{v-1}(x)\right. \\
&\left.\left.\left.\times f_{0, v}\left(a_{0, v}(x, y)\right) \cdots\right)\right)\right),
\end{aligned}
$$

where $a_{i, j}(x, y)=a_{i}(x)$ are univariate interpolating polynomial, rational interpolation, the Hermite interpolating polynomial, or Salzer-type osculatory rational interpolation. Then $\bar{Q}(x, y)$ is a general interpolation formula of block based univariate interpolation.

3.1.1. Special Case. We discuss the case that we choose the parameters in (2),

$$
\begin{aligned}
Q(x, y)=f_{0}\left(f_{0,0}(\right. & a_{0,0}(x, y) \\
& +g_{0}(x) \\
& \times f_{1,0}\left(a_{1,0}(x, y)+\cdots+g_{u-1}(x)\right. \\
& \left.\left.\left.\times f_{u, 0}\left(a_{u, 0}(x, y)\right) \cdots\right)\right)\right) .
\end{aligned}
$$

Some of the special cases of the above general interpolation formula of interpolation functions are shown below.

(1) If $f_{0}(x)=x, f_{i, 0}(x)=x, g_{s}(x)=\prod_{i=c_{s}}^{d_{s}}\left(x-x_{i}\right)$, $i=0,1, \ldots, n, s=0,1, \ldots, u-1$, then $\widetilde{Q}(x, y)$ is univariate block based Newton-like interpolation polynomial [12].

(2) If $f_{0}(x)=x, f_{0,0}(x)=x, f_{i, 0}(x)=1 / x, g_{s}(x)=$ $\prod_{i=c_{s}}^{d_{s}}\left(x-x_{i}\right), i=0,1, \ldots, n, s=0,1, \ldots, u-$ 1 , then $\widetilde{Q}(x, y)$ is univariate block based Thiele-like continued fractions interpolation [13].

(3) If $f_{0}(x)=x, f_{0,0}(x)=x, f_{i, 0}(x)=x^{(-1)^{i+1}}, g_{s}(x)=$ $\prod_{i=c_{s}}^{d_{s}}\left(x-x_{i}\right), i=0,1, \ldots, n, s=0,1, \ldots, u-1$, then $\widetilde{Q}(x, y)$ is univariate block based associated continued fractions interpolation [17].

(4) If $f_{0}(x)=x, f_{0,0}(x)=x, f_{i, 0}(x)=x$ or $1 / x, g_{s}(x)=$ $x-x_{s}, i=0,1, \ldots, n, s=0,1, \ldots, u-1$, then $\widetilde{Q}(x, y)$ is the general frame of interpolation scheme studied by Tan and Fang [1].

(5) If $f_{0}(x)=x, f_{0,0}(x)=x, f_{i, 0}(x)=x^{(-1)^{i+1}}, g_{s}(x)=$ $\prod_{i=c_{s}}^{d_{s}}\left(x-x_{i}\right), i=0,1, \ldots, n, s=0,1, \ldots, u-1$, then $\widetilde{Q}(x, y)$ is univariate block based associated continued fractions interpolation [17].
(6) If $f_{0}(x)=x, f_{0,0}(x)=x, f_{i, 0}(x)=x$ or $1 / x$, $i=0,1, \ldots, n$, then $\widetilde{Q}(x, y)$ is the general frame of interpolation scheme studied by Tang and Zou [17].

(7) If $f_{0}(x)=x$, then $\widetilde{Q}(x, y)$ is the general frames of interpolation scheme studied by Zou and Tang [20].

If we choose $d_{s}=c_{s}+1$ in scheme as shown above, that is to say, every block only includes one point, then $\widetilde{Q}(x, y)$ is changed into univariate Newton interpolation polynomial, Thiele continued fractions interpolation, and associated continued fractions interpolation. Furthermore, one can get some blending rational interpolations or osculatory interpolation via choosing $f_{i}, f_{i, j}$ appropriately; for example, one can get modified Thiele continued fractions blending rational interpolation, three associated continued fractions interpolation, block based Newton-Werner blending osculatory rational interpolation, Thiele-Werner blending osculatory rational interpolation, and so on $[16,24]$.

3.2. General Interpolation Formulae for Block Based Bivariate Blending Rational Interpolation. If we choose the parameters in formulae (2) and (25) as follows,

$$
\begin{gathered}
f_{1, i}(x) \equiv 0, \quad i=1,2, \ldots, n, \\
S_{s}(x, y)=\prod_{k=c_{s}}^{d_{s}}\left(x-x_{k}\right), \quad h_{t}(y)=\prod_{j=h_{t}}^{r_{t}}\left(y-y_{j}\right), \\
s=0,1, \ldots, u, t=0,1, \ldots v,
\end{gathered}
$$

we can get

$$
\begin{array}{r}
\widehat{Q}(x, y)=f_{0}\left(A_{0}(x, y)+S_{0}(x, y)\right. \\
\times f_{1}\left(A_{1}(x, y)+S_{1}(x, y)\right. \\
\times f_{2}\left(A_{2}(x, y)+\cdots+S_{u-1}(x, y)\right. \\
\left.\left.\left.\quad \times f_{u}\left(A_{u}(x, y)\right) \cdots\right)\right)\right) \\
\begin{array}{r}
A_{i}(x, y)=f_{i, 0}\left(a_{i, 0}(x, y)+h_{0}(y)\right. \\
\times f_{i, 1}\left(a_{i, 1}(x, y)+\cdots+h_{v-1}(y)\right. \\
\left.\left.\times f_{i, v}\left(a_{i, v}(x, y)\right) \cdots\right)\right) .
\end{array}
\end{array}
$$

then $\widehat{Q}(x, y)$ is a general interpolation formula of block based bivariate blending rational interpolation.

3.2.1. Special Case. Some of the special cases of the general interpolation formula of block based bivariate interpolation are shown below.

(1) If $f_{i}(x)=x, f_{i, j}(x)=x, i=0,1, \ldots, n, j=0,1, \ldots, m$, $S_{s}(x, y)=\prod_{k=c_{s}}^{d_{s}}\left(x-x_{k}\right), h_{t}(y)=\prod_{j=h_{t}}^{r_{t}}\left(y-y_{j}\right), s=$ $0,1, \ldots, u, t=0,1, \ldots v$, then $\widehat{Q}(x, y)$ is block based bivariate Newton-like blending rational interpolation 
[12]; especially, let $d_{s}=c_{s}+1$; that is to say, every block only includes one point; then, $\widehat{Q}(x, y)$ is bivariate Newton interpolation polynomial.

(2) If $f_{0}(x)=x, f_{i, 0}(x)=x, f_{i}(x)=1 / x, f_{i, j}(x)=1 / x$, $i=0,1, \ldots, n, j=0,1, \ldots, m, S_{s}(x, y)=\prod_{k=c_{s}}^{d_{s}}(x-$ $\left.x_{k}\right), h_{t}(y)=\prod_{j=h_{t}}^{r_{t}}\left(y-y_{j}\right), s=0,1, \ldots, u, t=$ $0,1, \ldots v$, then $\widehat{Q}(x, y)$ is block based bivariate Thielelike blending rational interpolation [13]; especially, let $d_{s}=c_{s}+1$; that is to say, every block only includes one point; then, $\widehat{Q}(x, y)$ is bivariate Thiele-type branched continued fractions interpolation $[11,25]$.

(3) If $f_{0}(x)=x, f_{i, 0}(x)=x, f_{i, j}(x)=x, f_{i}(x)=1 / x$, $i=0,1, \ldots, n, j=0,1, \ldots, m, S_{s}(x, y)=\prod_{k=c_{s}}^{d_{s}}(x-$ $\left.x_{k}\right), h_{t}(y)=\prod_{j=h_{t}}^{r_{t}}\left(y-y_{j}\right), s=0,1, \ldots, u, t=$ $0,1, \ldots v$, then $\widehat{Q}(x, y)$ is block based Newton-Thielelike blending rational interpolation [17]; especially, let $d_{s}=c_{s}+1$; that is to say, every block only includes one point; then, $\widehat{Q}(x, y)$ is bivariate Newton-Thiele blending rational interpolation $[11,20]$.

(4) If $f_{0}(x)=x, f_{i, 0}(x)=x, f_{i}(x)=x, f_{i, j}(x)=1 / x, i=$ $0,1, \ldots, n, j=0,1, \ldots, m, S_{s}(x, y)=\prod_{k=c_{s}}^{d_{s}}(x-$ $\left.x_{k}\right), h_{t}(y)=\prod_{j=h_{t}}^{r_{t}}\left(y-y_{j}\right), s=0,1, \ldots, u, t=$ $0,1, \ldots v$, then $\widehat{Q}(x, y)$ is block based Thiele-Newtonlike blending rational interpolation $[17,18]$; especially, let $d_{s}=c_{s}+1$; that is to say, every block only includes one point; then, $\widehat{Q}(x, y)$ is bivariate Thiele-Newton blending rational interpolation $[1,17,20]$.

(5) If $f_{0}(x)=x, f_{i}(x)=x^{(-1)^{i+1}}, f_{i, j}(x)=x, i=$ $0,1, \ldots, n, j=0,1, \ldots, m, S_{s}(x, y)=\prod_{k=c_{s}}^{d_{s}}(x-$ $\left.x_{k}\right), h_{t}(y)=\prod_{j=h_{t}}^{r_{t}}\left(y-y_{j}\right), s=0,1, \ldots, u, t=$ $0,1, \ldots v$, then $\widehat{Q}(x, y)$ is block based bivariate Newton associated continued fractions blending rational interpolation $[17,20]$; especially, let $d_{s}=c_{s}+1$; that is to say, every block only includes one point; then, $\widehat{Q}(x, y)$ is bivariate Newton associated continued fractions blending rational interpolation $[17,20,26]$.

(6) If $f_{i, 0}(x)=x, f_{i}(x)=x, f_{i, j}(x)=x^{(-1)^{i+1}}, i=$ $0,1, \ldots, n, j=0,1, \ldots, m, S_{s}(x, y)=\prod_{k=c_{s}}^{d_{s}}(x-$ $\left.x_{k}\right), h_{t}(y)=\prod_{j=h_{t}}^{r_{t}}\left(y-y_{j}\right), s=0,1, \ldots, u, t=$ $0,1, \ldots v$, then $\widehat{Q}(x, y)$ is block based bivariate associated continued fractions Newton blending rational interpolation $[17,20,26]$; especially, let $d_{s}=c_{s}+1$; that is to say, every block only includes one point; then, $\widehat{Q}(x, y)$ is bivariate associated continued fractions Newton blending rational interpolation $[17,20,26]$.

(7) If $f_{0}(x)=x, f_{i, 0}(x)=x, f_{i}(x)=1 / x$, $f_{i, j}(x)=x^{(-1)^{i+1}}, i=0,1, \ldots, n, j=0,1, \ldots, m$, $S_{s}(x, y)=\prod_{k=c_{s}}^{d_{s}}\left(x-x_{k}\right), h_{t}(y)=\prod_{j=h_{t}}^{r_{t}}\left(y-y_{j}\right)$, $s=0,1, \ldots, u, t=0,1, \ldots v$, then $\widehat{Q}(x, y)$ is block based bivariate Thiele associated continued fractions blending rational interpolation $[17,20,26]$; especially, let $d_{s}=c_{s}+1$; that is to say, every block only includes one point; then, $\widehat{Q}(x, y)$ is bivariate Thiele associated continued fractions blending rational interpolation $[17,20,26]$.

(8) If $f_{0}(x)=x, f_{i, 0}(x)=x, f_{i}(x)=x^{(-1)^{i+1}}, f_{i, j}(x)=$ $1 / x, i=0,1, \ldots, n, j=0,1, \ldots, m, S_{s}(x, y)=$ $\prod_{k=c_{s}}^{d_{s}}\left(x-x_{k}\right), h_{t}(y)=\prod_{j=h_{t}}^{r_{t}}\left(y-y_{j}\right), s=0,1, \ldots, u$, $t=0,1, \ldots v$, then $\widehat{Q}(x, y)$ is block based bivariate associated continued fractions Thiele blending rational interpolation $[17,20,26]$; especially, let $d_{s}=$ $c_{s}+1$; that is to say, every block only includes one point; then, $\widehat{Q}(x, y)$ is bivariate associated continued fractions Thiele blending rational interpolation [17, 20, 26].

(9) If $f_{0}(x)=x, f_{i, 0}(x)=x, f_{i}(x)=x^{(-1)^{i+1}}, f_{i, j}(x)=$ $x^{(-1)^{i+1}}, i=0,1, \ldots, n, j=0,1, \ldots, m, S_{s}(x, y)=$ $\prod_{k=c_{s}}^{d_{s}}\left(x-x_{k}\right), h_{t}(y)=\prod_{j=h_{t}}^{r_{t}}\left(y-y_{j}\right), s=0,1, \ldots, u$, $t=0,1, \ldots v$, then $\widehat{Q}(x, y)$ is block based bivariate associated continued fractions blending rational interpolation $[17,20,26]$; especially, let $d_{s}=c_{s}+1$; that is to say, every block only includes one point; then, $\widehat{Q}(x, y)$ is bivariate branched associated continued fractions blending rational interpolation $[17,20,26$, 27].

(10) If $f_{i}(x)=x, f_{i, j}(x)=x, u=n, S_{s}(x)=\sin x-\sin x_{i}$, $h_{j}(y)=\sin y-\sin y_{j}, i=0,1, \ldots, n ; j=0,1, \ldots, m$, then $\widehat{Q}(x, y)$ is a bivariate trigonometric function and may be expanded to a finite Fourier series.

(11) If we set $u=n, S_{s}(x)=s(x)-s\left(x_{i}\right), h_{j}(y)=s(y)-$ $s\left(y_{j}\right)$ and choose $f_{i, j}(x), f_{i}(x), i=0,1, \ldots, n, j=$ $0,1, \ldots, m$, from $x, 1 / x ; s(x), s(y)$ are chosen from $e^{x}, x^{2}, \cos x, e^{-x}, \operatorname{sh} x$, and so forth, then we have a class of interpolation functions.

(12) If $f_{0}(x)=x, f_{i}(x)=x$, or $f_{i}(x)=1 / x, f_{i, j}(x)=x$, or $f_{i, j}(x)=1 / x, u=n, v=m, S_{s}(x, y)=\left(x-x_{s}\right)$, $h_{t}(y)=\left(y-y_{j}\right), i=0,1, \ldots, n, j=0,1, \ldots, m$, then $\widehat{Q}(x, y)$ is the general frame of interpolation scheme studied by Tan and Fang [1].

(13) If $f_{0}(x)=x, f_{i}(x)=x^{(-1)^{i+1}}, f_{i, j}(x)=x^{(-1)^{i+1}}, u=n$, $v=m, S_{s}(x, y)=\left(x-x_{s}\right), h_{t}(y)=\left(y-y_{j}\right), i=$ $0,1, \ldots, n, j=0,1, \ldots, m$, then $\widehat{Q}(x, y)$ is the general frame of interpolation scheme studied by Tang and Zou [17].

(14) If $f_{0}(x)=x, f_{i}(x)=x^{(-1)^{i+1}}, f_{i, j}(x)=x^{(-1)^{i+1}}, i=$ $0,1, \ldots, n, j=0,1, \ldots, m$, then $\widehat{Q}(x, y)$ is the general frame of interpolation scheme studied by Tang and Zou [17].

(15) If $S_{s}(x, y), h_{t}(y), s=0,1, \ldots, u, t=0,1, \ldots, v$, are the same as $g_{i}(x), h_{i, j}(y), i=0,1, \ldots, n, j=0,1, \ldots, m$, of paper [20], then $\widehat{Q}(x, y)$ is the general frame of interpolation scheme studied by Zou and Tang [20]. 
Special cases (10) and (11) are interesting and useful; we will investigate them in depth in the future. Furthermore, one can get more blending rational interpolations via choosing $f_{i}(x), f_{i, j}(x)$ appropriately. It could be used to deal with the interpolation problems where inverse differences are nonexistent or unattainable points occur via choosing $f_{i}(x), f_{i, j}(x)$ appropriately [17].

3.3. Algorithm of General Interpolation Formulae of Block Based Bivariate Interpolation. In this section, we give the algorithm of general interpolation formula of block based bivariate interpolation.

$f_{i}, f_{i, j}$ given is initialized.

Step 1. Let

$$
f(x, y)=f_{i, j}^{0,0}, \quad i=0,1, \ldots, n, j=0,1, \ldots, m .
$$

Step 2. For $t=1,2, \ldots, v$,

$$
\begin{aligned}
f_{i, j}^{0, t}= & f_{0, t}^{-1}\left(\frac{f_{i, j}^{0, t-1}-a_{0, t-1}\left(x_{i}, y_{j}\right)}{h_{t-1}\left(y_{j}\right)}\right), \\
& i=0,1, \ldots, n, j=h_{t}, h_{t}+1, \ldots, m .
\end{aligned}
$$

Step 3. For $s=1,2, \ldots, u$,

$$
\begin{aligned}
f_{i, j}^{s, 0} & =f_{s}^{-1}\left(\frac{f_{i, j}^{s-1,0}-Z_{s-1}\left(x_{i}, y_{j}\right)}{S_{s-1}\left(x_{i}\right)}\right), \\
i & =c_{s}, c_{s}+1, \ldots, n ; j=0,1, \ldots, m .
\end{aligned}
$$

Step 4. For $s=1,2, \ldots, u, t=1,2, \ldots, v$,

$$
\begin{aligned}
f_{i, j}^{s, t}= & f_{s, t}^{-1}\left(\frac{f_{i, j}^{s, t-1}-a_{s, t-1}\left(x_{i}, y_{j}\right)}{h_{t-1}\left(y_{j}\right)}\right), \\
& i=c_{s}, c_{s}+1, \ldots, n ; j=h_{t}, h_{t}+1, \ldots, m .
\end{aligned}
$$

Step 5. For $s=1,2, \ldots, u, t=1,2, \ldots, v$,

$$
\begin{gathered}
a_{s, t}(x, y)=f_{i, j}^{s, t}, \quad c_{s} \leq i \leq d_{s}, \quad h_{t} \leq j \leq r_{t}, \\
s=0,1, \ldots, u, t=0,1, \ldots, v,
\end{gathered}
$$

where $a_{s, t}(x, y)(t=0,1, \ldots, v)$ are bivariate polynomials or rational interpolations on the subsets $\prod_{n, m}^{s, t}$.

3.4. Dual Scheme for General Interpolation Formulae for Block Based Bivariate Interpolation. If we choose the parameters in formulae (2) and (25) as follows,

$$
\begin{gathered}
f_{i, 1}(x) \equiv 0, \quad S_{t}(x, y)=\prod_{j=h_{t}}^{r_{t}}\left(y-y_{j}\right), \\
g_{s}(x)=\prod_{k=c_{s}}^{d_{s}}\left(x-x_{k}\right), \quad s=0,1, \ldots, u, t=0,1, \ldots v,
\end{gathered}
$$

TABLE 1: Interpolation data.

\begin{tabular}{cccc}
\hline & $x_{0}=0$ & $x_{1}=1$ & $x_{2}=2$ \\
\hline$y_{0}=0$ & 1 & 2 & 4 \\
$y_{1}=1$ & 0 & 3 & 5 \\
$y_{2}=2$ & -2 & -3 & 2 \\
\hline
\end{tabular}

then

$$
\begin{aligned}
\vec{Q}(x, y)=f_{0}\left(A_{0}(x, y)\right. & +S_{0}(x, y) \\
\times f_{1}\left(A_{1}(x, y)+S_{1}(x, y)\right. & \\
& \times f_{2}\left(A_{2}(x, y)+\cdots+S_{v-1}(x, y)\right. \\
& \left.\left.\times f_{v}\left(A_{v}(x, y)\right) \cdots\right)\right), \\
A_{i}(x, y)=f_{0, i}\left(a_{0, i}(x, y)\right. & +g_{0}(x) \\
\times f_{1, i}\left(a_{1, i}(x, y)+\cdots+g_{u-1}(x)\right. & \\
& \left.\left.\times f_{u, i}\left(a_{u, i}(x, y)\right) \cdots\right)\right) .
\end{aligned}
$$

We call the scheme defined by formulae (41)-(42) dual scheme of general interpolation formula for block based bivariate interpolation of (34)-(35). We can discuss this frame similarly, and the above dual interpolation function also includes many kinds of the interpolation schemes which does not as the same as the schemes we have discussed in Section 3.2.

\section{Numerical Examples}

In this section, we take two simple examples to show the effectiveness of the result in this paper. Example 4 is to show how the proposed construction takes out under different choice of $f_{i}$ 's, $f_{i, j}$ 's and $g$ 's, $h$ 's. Example 10 is given to solve the interpolation problem where inverse differences are nonexistent.

Example 4. Let $\left(x_{i}, y_{j}\right)$ and $f\left(x_{i}, y_{j}\right)(i=0,1,2, j=0,1,2)$ be given in Table 1 .

Using the frame in the paper, one can get many special interpolations; some of them are as follows.

Scheme 1. Symmetric continued fractions interpolation is

$$
\begin{aligned}
Q_{1}(x, y)=1 & +\frac{x}{1+\frac{x-1}{1}}+\frac{y}{-1+\frac{y-1}{2}} \\
& +\frac{x y}{1 / 2+((y-1) /(-2 / 5))}+\frac{(x-1)(y-1)}{1 / 3} .
\end{aligned}
$$


Scheme 2. Bivariate Newton interpolation polynomial is

$$
\begin{aligned}
Q_{2}(x, y)= & 1-y-\frac{1}{2} y(y-1)+x \\
& +\frac{1}{2} x(x-1)+2 x y-3 x y(y-1) \\
& -x y(x-1)+\frac{9}{4} x(x-1) y(y-1) .
\end{aligned}
$$

Scheme 3. Bivariate Thiele branched continued fractions interpolation is

$$
\begin{aligned}
Q_{3}(x, y)= & +\frac{y}{-1+\frac{y-1}{3}} \\
& +\frac{x}{1+(y /((-3 / 2)+(y-1) / 2))} \\
& +\frac{x-1}{-3+(y /((1 / 18)+(y-1) /(198 / 97)))} .
\end{aligned}
$$

Scheme 4. Bivariate Newton-Thiele blending rational interpolation is

$$
\begin{aligned}
Q_{4}(x, y)= & 1-y-\frac{1}{2} y(y-1) \\
& +\frac{x}{1-(2 y / 3)-(y(y-1) / 3)} \\
& +\frac{x-1}{-3+18 y-(97 / 6) y(y-1)}
\end{aligned}
$$

Scheme 5. Bivariate Thiele-Newton blending rational interpolation is

$$
\begin{aligned}
Q_{5}(x, y)= & 1+\frac{y}{-1+\frac{y-1}{3}}+\left(1+\frac{y}{1 / 2+\frac{y-1}{-2 / 3}}\right) x \\
& +\left(\frac{1}{2}+\frac{y}{-1+\frac{y-1}{5 / 9}}\right) x(x-1) .
\end{aligned}
$$

If $c_{0}=0, d_{0}=1, c_{1}=d_{1}=2 ; h_{0}=0, r_{0}=1, h_{1}=r_{1}=$ 2 , namely, we divided $\prod_{2,2}$ into the following four subsets $\prod_{2,2}^{0,0}, \prod_{2,2}^{0,1}, \prod_{2,2}^{1,0}$, and $\prod_{2,2}^{1,1}$ :

$$
\begin{array}{lll}
(0,0) & (0,1) & (0,2) \\
(1,0) & (1,1) & (1,2) \\
(2,0) & (2,1) & (2,2)
\end{array}
$$

Let $a_{0,0}$ be bivariate Newton interpolating polynomial $\prod_{2,2}^{0,0}, a_{0,1}$ bivariate Newton interpolating polynomial $\prod_{2,2}^{0,1}$, $a_{1,0}$ bivariate Newton interpolating polynomial $\prod_{2,2}^{1,0}$, and $a_{1,1}$ bivariate Newton interpolating polynomial $\prod_{2,2}^{1,1}$.
TABLE 2: Interpolation data.

\begin{tabular}{cccc}
\hline & $x_{0}=0$ & $x_{1}=1$ & $x_{2}=2$ \\
\hline$y_{0}=0$ & 1 & 3 & 4 \\
$y_{1}=1$ & 0 & 1 & 1 \\
$y_{2}=2$ & -2 & -3 & -1 \\
\hline
\end{tabular}

Scheme 6. Block based bivariate Thiele-Newton blending rational interpolation is

$$
\begin{aligned}
Q_{6}(x, y)= & +x-y+2 x y+\frac{y(y-1)}{-2+12 x / 7} \\
& +\left[\frac{1}{2}-y+\frac{y(y-1)}{-20 / 27}\right] x(x-1) .
\end{aligned}
$$

Scheme 7. Block based bivariate Newton-Thiele blending rational interpolation is

$$
\begin{aligned}
Q_{7}(x, y)= & 1+x-y+2 x y+\left(-\frac{1}{2}-3 x\right) y(y-1) \\
& +\frac{x(x-1)}{2-4 y+(19 / 6) y(y-1)} .
\end{aligned}
$$

Scheme 8. Block based bivariate Newton-like blending rational interpolation is

$$
\begin{aligned}
Q_{8}(x, y)= & 1+x-y+2 x y+\left(-\frac{1}{2}-3 x\right) y(y-1) \\
& +\left(\frac{1}{2}-y+\frac{9}{4} y(y-1)\right) x(x-1) .
\end{aligned}
$$

Scheme 9. Block based bivariate Thiele-like blending rational interpolation is

$$
\begin{aligned}
Q_{9}(x, y)= & 1+x-y+2 x y \\
& +\frac{y(y-1)}{-2+(12 x / 7)} \\
& +\frac{x(x-1)}{2-4 y+(y(y-1) /(42 / 121))} .
\end{aligned}
$$

It is easy to verify

$$
\begin{aligned}
Q_{s}\left(x_{i}, y_{j}\right) & =f\left(x_{i}, y_{j}\right), \\
i & =0,1,2 ; \quad j=0,1,2, s=1,2, \ldots, 9 .
\end{aligned}
$$

Example 10. Let $\left(x_{i}, y_{j}\right)$ and $f\left(x_{i}, y_{j}\right)$ be given in Table 2 .

Newton-Thiele blending rational interpolation fails in this case, since calculating inverse differences leads to that 
one of denominator is zero. From the general frame (34)-(35), by choosing $f_{i}(x), f_{i, j}(x)$ appropriately, we can get

$$
\begin{aligned}
& L_{1}=1+\frac{y}{-1+(y-1) / 3}, \\
& L_{2}=2+\frac{y}{-1+(y-1) / 3}, \\
& L_{3}=\frac{-1}{2}+y(y-1)
\end{aligned}
$$

and we can get the following interpolation function:

$$
\begin{aligned}
Q_{2,2} & (x, y) \\
= & L_{1}+x L_{2}+x(x-1) L_{3} \\
= & \left(8 y-8+3 x y-20 x+7 x^{2} y+4 x^{2}\right. \\
& \left.\quad+2 x^{2} y^{3}-10 x^{2} y^{2}-2 x y^{3}+10 x y^{2}\right) \\
& \quad \times(2 y-8)^{-1} .
\end{aligned}
$$

It is easy to verify

$$
Q_{2,2}\left(x_{i}, y_{j}\right)=f\left(x_{i}, y_{j}\right), \quad i=0,1,2 ; j=0,1,2 .
$$

\section{Conclusion}

The general interpolation formulae of bivariate interpolation function are more general than the general frames studied by many scholars $[1,14-20]$; it could be used to deal with the interpolation problems where inverse differences are nonexistent or unattainable points occur via choosing $f_{i}$, $f_{i, j}$ appropriately [17]. Another question is coming; there are so many schemes we can use; how to choose formula appropriately is our further work. In practical applications, the choice of $f_{i}$ 's, $f_{i, j}$ 's and $g$ 's, $h$ 's may be determined by the desired form of interpolation, for example, polynomial, rational function of given degree of the numerator and the denominator, or certain transformation of a rational function. If there is no restriction as to the form of $Q(x, y)$, the best choice may be the interpolation function that gives the smallest error term among the functions certain complexity. However, it is not easy to determine such a function without the process of trial and comparison.

We conclude this paper by pointing out that it is not difficult to generalize the general interpolation formulae in this paper to rational interpolation for higher dimensions, vector-valued case, or matrix-valued case $[16,17,22,23]$.

\section{Conflict of Interests}

The authors declare that there is no conflict of interests regarding the publication of this paper.

\section{Acknowledgment}

This work is supported by the Grants of the National Natural Science Foundation of China, nos. 61005010 and 61272024, and the Anhui Provincial Natural Science Foundation, nos. 1308085MF84 and 1308085QF115.

\section{References}

[1] J.-Q. Tan and Y. Fang, "General frames for bivariate interpolation," Journal of Mathematical Research and Exposition, vol. 19, no. 4, pp. 681-687, 1999.

[2] A. Cuyt and B. Verdonk, "Multivariate rational interpolation," Computing, vol. 34, no. 1, pp. 41-61, 1985.

[3] A. Cuyt and B. Verdonk, "Multivariate reciprocal differences for branched Thiele continued fraction expansions," Journal of Computational and Applied Mathematics, vol. 21, no. 2, pp. 145160, 1988.

[4] H. I. Kučminskaja, "Approximation of functions by continued and branching continued fractions," Matematicheskie Metody $i$ Fiziko-Mekhanicheskie Polya, no. 12, pp. 3-10, 1980.

[5] J. A. Murphy and M. R. O'Donohoe, "A two-variable generalization of the Stieltjes-type continued fraction," Journal of Computational and Applied Mathematics, vol. 4, no. 3, pp. 181190, 1978

[6] K. Kuchmins'ka and S. Vozna, "On Newton-Thiele-like interpolating formula," Communications in the Analytic Theory of Continued Fractions, vol. 8, pp. 74-79, 2000.

[7] Kh. I. Kuchmins'ka, O. M. Sus, and S. M. Vozna, "Approximation properties of two-dimensional continued fractions," Ukrainian Mathematical Journal, vol. 55, no. 1, pp. 30-44, 2003.

[8] M. M. Pahirya and T. S. Svyda, "Problem of interpolation of functions by two-dimensional continued fractions," Ukrainian Mathematical Journal, vol. 58, no. 5, pp. 954-966, 2006.

[9] Q.-J. Zhao and J.-Q. Tan, "The limiting case of blending differences for bivariate blending continued fraction expansions," Northeastern Mathematical Journal, vol. 22, no. 4, pp. 404-414, 2006.

[10] J. Z. Wang, “Stieltjes-Newton's rational interpolants," Communication on Applied Mathematics and Computation, vol. 20, no. 2, pp. 77-82, 2006.

[11] R.-H. Wang and J. Qian, "Bivariate polynomial and continued fraction interpolation over ortho-triples," Applied Mathematics and Computation, vol. 217, no. 19, pp. 7620-7635, 2011.

[12] Q.-J. Zhao and J.-Q. Tan, "Block based Newton-like blending interpolation," Journal of Computational Mathematics, vol. 24, no. 4, pp. 515-526, 2006.

[13] Q.-J. Zhao and J. Tan, "Block-based Thiele-like blending rational interpolation," Journal of Computational and Applied Mathematics, vol. 195, no. 1-2, pp. 312-325, 2006.

[14] S. W. Kahng, Generalized Newton's Interpolation Functions and Their Applications to Chebyahev Approximations, Lockheed Electronics Company Report, 1967.

[15] S. W. Kahng, "Osculatory interpolation," Mathematics of Computation, vol. 23, pp. 621-629, 1969.

[16] J. Q. Tan, Theory of Continued Fractions and Its Applications, Science Publishers, Beijing, China, 2007, (Chinese).

[17] S. Tang and L. Zou, "A note on general frames for bivariate interpolation," Journal of Mathematical Research and Exposition, vol. 29, no. 4, pp. 700-706, 2009. 
[18] C. W. Li, X. L. Zhu, W. R. Lin, and H. H. Chen, "Blockbased bivariate blending rational interpolation," Journal of Hefei University of Technology, vol. 31, no. 3, pp. 484-488, 2008.

[19] L. Zou and S. Tang, "Notes on general frames of symmetric interpolation," Communication on Applied Mathematics and Computation, vol. 25, no. 1, pp. 111-118, 2011.

[20] L. Zou and S. Tang, "General structures of block based interpolational function," Communications in Mathematical Research, vol. 28, no. 3, pp. 193-208, 2012.

[21] H. E. Salzer, "Some new divided difference algorithm for two variables," in On Numerical Approximation, R. E. Langer, Ed., The University of Wisconsin Press, Madison, Wis, USA, 1959.

[22] J. Q. Tan and G. Q. Zhu, "General framework for vector-valued interpolants," in Proceedings of the 3rd China-Japan Seminar on Numerical Mathematics, Z. Shi, Ed., pp. 273-278, Science Press, 1998.

[23] G.-Q. Zhu and J.-Q. Tan, "A note on matrix-valued rational interpolants," Journal of Computational and Applied Mathematics, vol. 110, no. 1, pp. 129-140, 1999.

[24] J. B. Wang and C. Q. Gu, "Vector valued Thiele-Werner-type osculatory rational interpolants," Journal of Computational and Applied Mathematics, vol. 163, no. 1, pp. 241-252, 2004.

[25] W. Siemaszko, "Thiele-type branched continued fractions for two-variable functions," Journal of Computational and Applied Mathematics, vol. 9, no. 2, pp. 137-153, 1983.

[26] L. Zou and S. Tang, "New approach to bivariate blending rational interpolants," Chinese Quarterly Journal of Mathematics, vol. 26, no. 1, pp. 280-284, 2011.

[27] S. M. Vozna, "Newton-Thiele-type interpolational formula in the form of two dimensional continued fraction with non-equivalent variables," Matematicheskie Metody i FizikoMekhanicheskie Polya, vol. 47, pp. 67-72, 2004. 


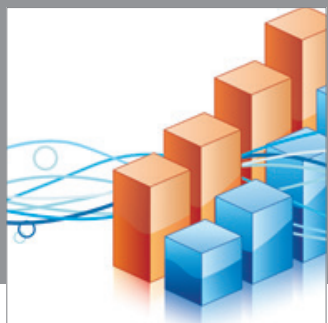

Advances in

Operations Research

mansans

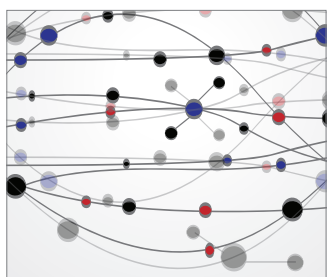

The Scientific World Journal
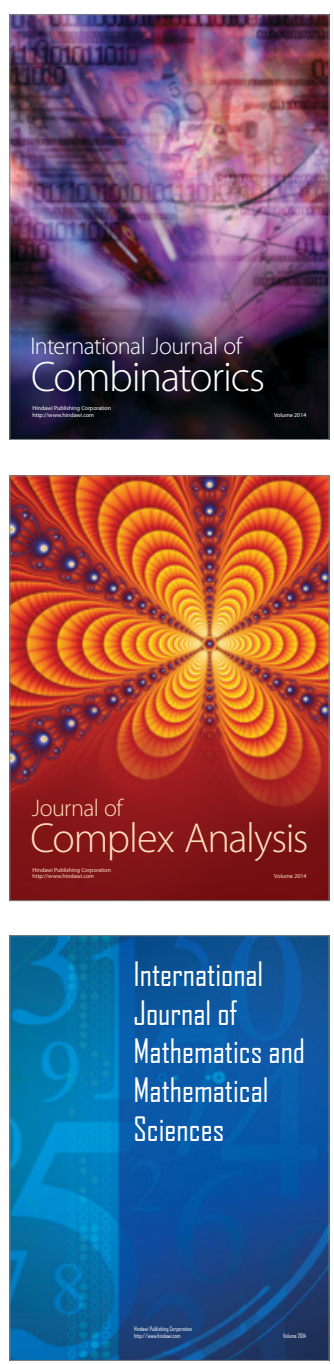
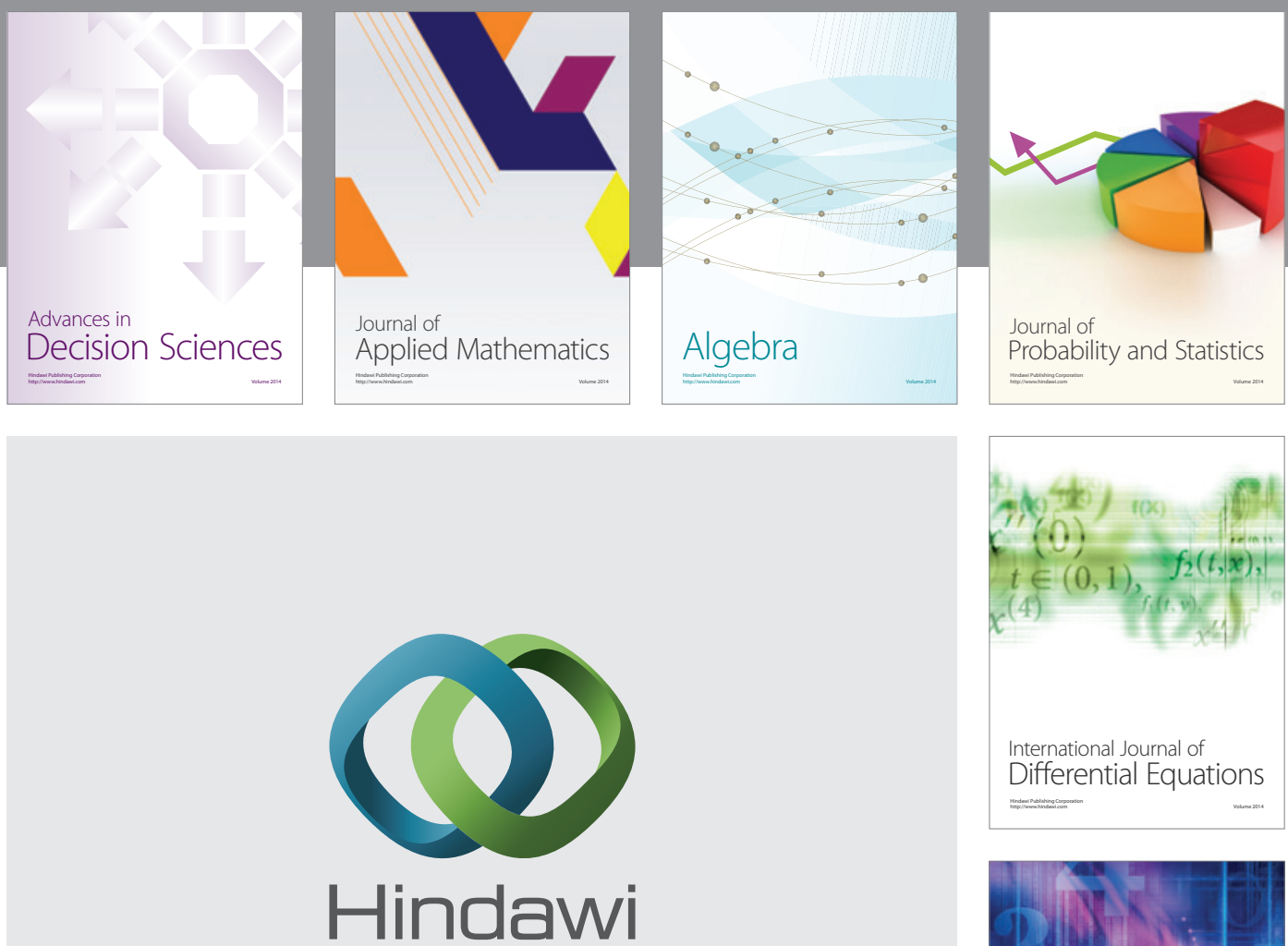

Submit your manuscripts at http://www.hindawi.com
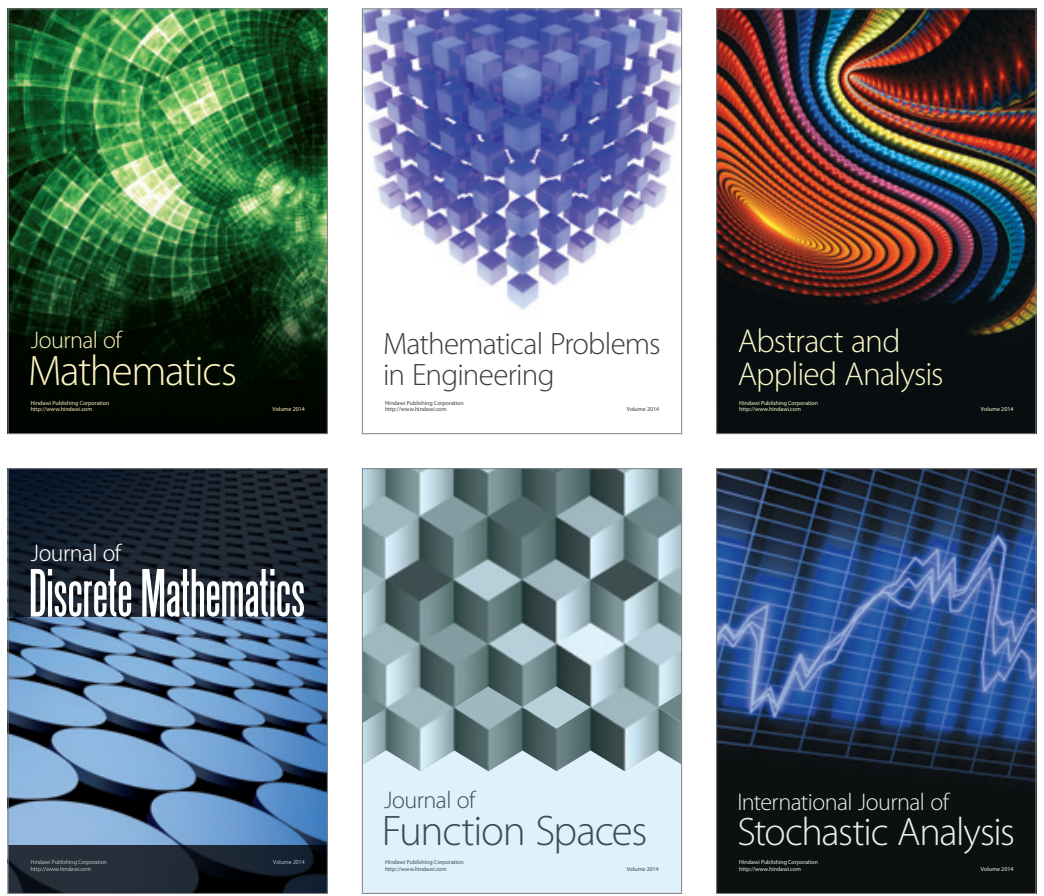

Journal of

Function Spaces

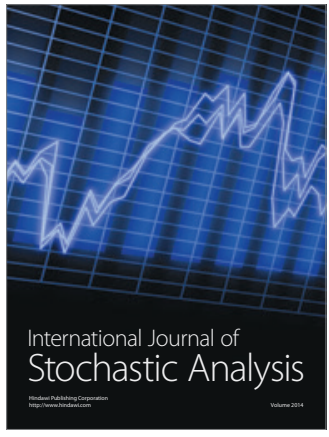

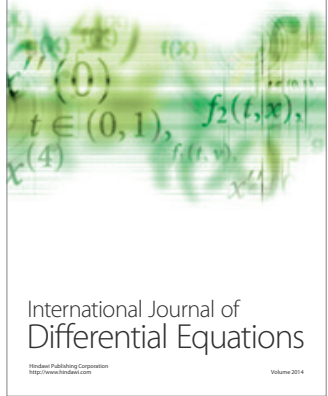
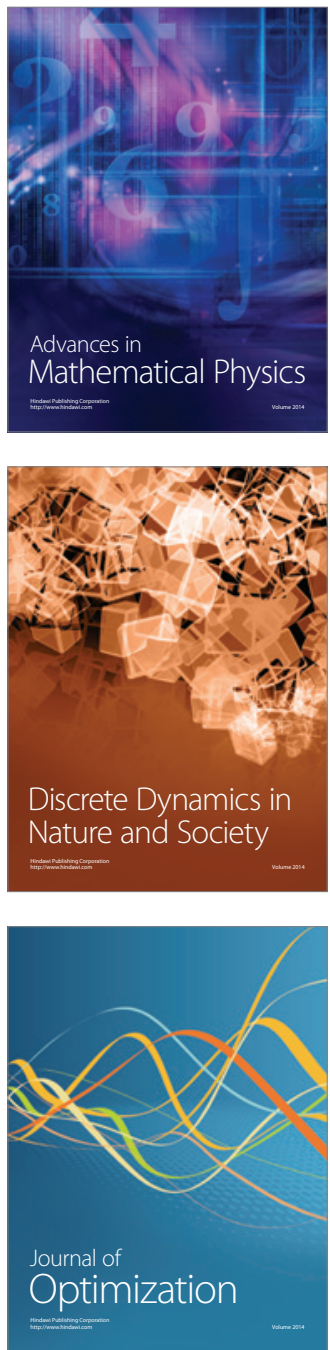